Mots. Les langages du politique

\title{
Publicité sans frontières. De la pub au politique
}

\section{Benjamin Delalande}

\section{CpenEdition}

Journals

Édition électronique

URL : http://journals.openedition.org/mots/20613

DOI : $10.4000 /$ mots. 20613

ISSN : 1960-6001

\section{Éditeur}

ENS Éditions

\section{Édition imprimée}

Date de publication : 1 mai 2012

Pagination : 79-94

ISBN : 978-2-84788-356-5

ISSN : 0243-6450

Référence électronique

Benjamin Delalande, "Publicité sans frontières. De la pub au politique », Mots. Les langages du politique [En ligne], 98 | 2012, mis en ligne le 01 mai 2014, consulté le 01 mai 2019. URL : http:// journals.openedition.org/mots/20613; DOI : 10.4000/mots.20613 


\section{Publicité sans frontières. De la pub au politique}

Durant l'année 2005, le géant de la distribution E. Leclerc recycle toute une iconographie contestataire des mouvements de Mai 68 et des révolutions sociales de la fin des années 1960 pour produire des campagnes publicitaires institutionnelles ${ }^{1}$. Un seul message : défendre le pouvoir d'achat des consommateurs en dénonçant la hausse des prix. Mais en creux, l'entreprise dénonce la loi sur la loyauté et l'équilibre des relations commerciales (ou loi Galland) qui prévoit la réglementation des relations entre la grande distribution et ses fournisseurs. Le discours publicitaire qui est construit à partir d'une symbolique visuelle déjà fortement ancrée dans le champ social, réactualise une posture militante.

À partir d'une étude de cas, cet article propose d'interroger les liens entre le discours publicitaire et le politique en mobilisant les outils de la sémiotique (Greimas, Courtés, 1993; Rastier, 1987). Ces liens sont travaillés à partir de la notion de politisation (Achache, 1990 ; Arnaud, Guionnet, 2005; Bacot, 1991, 1993) et de la réflexion de Dominique Quessada (1999) qui pose que le discours publicitaire - en tant que discours qui dissout des frontières, dont celles de sa propre activité - engendre une incertitude entre ce qui est politique et ce qui ne l'est pas. En effet, selon lui, le discours publicitaire devient lui-même un discours politique qui ne relève plus de l'ordre du politique alors qu'il prétend s'y substituer. Dès lors, comment penser la communication publicitaire des entreprises quand celles-ci prétendent défendre l’intérêt général?

1. Depuis 1999, c'est à l'agence Australie que E. Leclerc a confié son budget national de communication. Les publicités du corpus couvrent la période 2004-2008; elles ont fait l'objet de campagnes d'affichage nationales et de campagnes de presse. 


\title{
"Acheter le moins cher possible pour vendre le moins cher possible " : le système E. Leclerc
}

\author{
Le pouvoir d'achat et la loi Galland
}

En 1949, Édouard Leclerc réinvente le commerce en appliquant une idée simple : «Acheter le moins cher possible pourvendre le moins cher possible ». La stratégie commerciale consiste à vendre des produits de consommation courante à prix de gros en s'approvisionnant directement chez les producteurs pour diminuer le nombre des intermédiaires. En réduisant les prix, l'enseigne connaît alors un succès commercial et un développement sans précédent qui transforment, en quelques années, la petite épicerie de Landerneau en une chaîne de grandes surfaces, numéro un de la grande distribution en France (FCA, 2011).

Depuis l'apparition des hypermarchés en France dans les années 1960, l'histoire des centres $\mathrm{E}$. Leclerc est associée à des prises de position contre les atteintes portées au droit de la concurrence, de la libre entreprise et du libre choix des consommateurs. Adapter et valoriser une politique commerciale par une communication publicitaire qui fait écho à l'actualité, voilà le principe essentiel sur lequel Leclerc règle sa conduite. En effet, à la suite du choc pétrolier de 1973 apparaissent des stations-service E. Leclerc qui proposent des prix sur les carburants moins élevés qu'ailleurs; en 1981, alors que le ministre de la Culture Jack Lang impose le livre à prix unique, l'enseigne outrepasse la loi en affichant une réduction de $20 \%$, ce qui lui permet de devenir en quelques années le deuxième libraire de France; E. Leclerc est également le premier grand distributeur à supprimer les sacs plastique gratuits de ses caisses pour participer à la protection de l'environnement.

En poursuivant l'engagement historique de son père, Michel-Édouard Leclerc fait de la défense du pouvoir d'achat un thème récurrent de communication qui engage l'enseigne durablement auprès de ses clients. Dans un contexte de crise économique, les campagnes publicitaires montrent ce positionnement comme un principe fédérateur (Hébert, 1993). Selon Michel-Édouard Leclerc, " [la démocratisation de] l'accès à un maximum de biens de consommation et de services» serait une nécessité pour défendre le pouvoir d'achat mais elle en serait empêchée par la loi Galland, votée en 1996, qui interdit « aux distributeurs de répercuter sur les prix de vente les avantages et remises négociés auprès des industriels» (Leclerc, 2008). Depuis son entrée en vigueur en 1997, l'enseigne milite donc pour une réforme de la loi afin d'améliorer, dit-elle, les relations commerciales entre fournisseurs et distributeurs et ainsi de faire baisser les prix à la consommation. Mais si l'on suit l'analyse de Perona (2004), «la loi Galland n'empêche pas les prix de baisser. Elle donne plus de pouvoir de négociation aux fournisseurs au détriment des distributeurs ». 


\section{Vers une mobilisation de la notion de politisation}

L'analyse du corpus va interroger cette question du pouvoir d'achat dans la construction du discours publicitaire de E. Leclerc à travers des campagnes, dites institutionnelles, qui ne valorisent aucun produit mais qui cherchent à installer une légitimité en énonçant un discours destiné à l'opinion publique. Selon Gilles Achache, quand l'entreprise fait valoir cette légitimité, elle tient un discours qui n'est pas celui «d'un acteur économique intervenant sur un marché poury vendre ses produits, mais [celui] d'un acteur civique situant son action dans l'horizon de l'intérêt général »; il s'agit d'un processus qu'il qualifie de «politisation» et qui demande l'approbation par l'opinion de l'action menée par l'entreprise (Achache, 1990).

Dans cette perspective, E. Leclerc emprunterait le rôle d'un acteur civique situant son intérêt commercial dans celui de l'intérêt général. Pour autant, peuton parler de "politisation » comme le fait Achache et qu'entend-on par cette notion? La question complexe du politique amène certains auteurs à ne pas le définir : Nina Eliasoph évoque un «discours orienté vers l'esprit public »², lequel se caractérise par son ouverture au débat en abordant des questions relevant du bien commun.

Pour sa part, Paul Bacot considère

[...] qu'il n'y a passage au politique que lorsque est opérée une mise en relation entre les visions opposées d'un problème et de sa solution, et les visions opposées d'autres problèmes et de leur solution [...]. On peut parler de traduction d'un clivage dans un autre clivage, ou mieux, d' « intertraduction » de clivages mis en équivalence. La problématisation ainsi élargie, la question est déplacée. Les problèmes sont reliés entre eux : la politisation est un processus caténaire. Le travail politique est donc globalisation et généralisation. Il apporte de la transversalité et transgresse les frontières [...]. (Bacot, 1991, p. 87)

La politisation est ainsi une construction sociale de la conflictualité ou conflictualisation. Sans cette dernière, il n'y a donc pas de politisation; néanmoins la conflictualisation sans politisation reste possible. À partir de l'analyse discursive de campagnes publicitaires, l'objectif de l'article est d'analyser de quelle manière le discours publicitaire entretient des liens avec la dimension politique.

\section{De la conflictualisation dans le discours publicitaire?}

En 2004, après avoir créé avec le BIPE3 un indicateur du «pouvoir d'achat effectif du consommateur» montrant une baisse de $1 \%$ (Mauriac, Nathan,

2. Eliasoph (1998), citée par Hamidi (2006).

3. Le BIPE est une société d'études économiques et de conseil en stratégie. 
2004), E. Leclerc lance une campagne pour contester l'augmentation de 1,2 \% du pouvoir d'achat annoncée par les chiffres officiels de l'INSEE (figure 1) 4.

Pugnace et provoquant, le distributeur sort en 2007 une nouvelle campagne incisive qui pourrait même irriter en haut lieu en s'adressant à « Monsieur le Président» (figures 2 et 3). Les énoncés textuels produisent ici une structure dialogique apparente qui articule le conflit entre l'énonciateur (E. Leclerc) et l'énonciataire («Monsieur le Président ») en reprochant à ce dernier de ne pas tenir la promesse de la campagne présidentielle de 2007 concernant l'augmentation du pouvoir d'achat. Constatant cet échec, E. Leclerc déclare que si hausse il y a, elle ne concerne que les prix. En effet, le camembert «Président » augmente de $18,8 \%$ quand les sondages annoncent une baisse de la popularité du chef de l'État (Jeudy, 2008). Interpeller le président et lui signaler un début difficile («c'est mal parti ») revient à faire le bilan d'une action politique sur la base d'un programme électoral. L'expression d'une telle opinion ne trouve habituellement pas sa place dans la communication publicitaire d'une entreprise privée.

Cependant, une publicité ne se réduit pas uniquement à sa structure textuelle. L'assemblage du textuel et de l'iconique produit des discours complexes dans lesquels interagissent différents langages, ce qui donne aux discours publicitaires une structure sémiotique mixte et instable, variant avec le dosage entre le texte et l'image. Cette mixité, produite par l'articulation des différentes composantes, bascule au profit de l'iconique pour capter le réel. Les analyses suivantes tentent de mettre au jour les stratégies énonciatives qui recourent au détournement d'une iconographie fortement ancrée par sa dimension politique.

\section{Recyclage de l'iconographie contestataire}

\section{Mai 68 au service du discours publicitaire}

À l'automne 2003, des actions de contestation de la publicité dans le métro parisien médiatisent le «mouvement antipub» qui détourne, à des fins critiques, les affiches publicitaires selon un principe post-situationniste et sur un mode graphique rappelant certains aspects de ce qui se faisait en Mai 68. Tout en poursuivant son combat pour le pouvoir d'achat, E. Leclerc franchit une étape supplémentaire en reprenant à son tour des symboles contestataires, dans une campagne parue pendant l'hiver 2005 qui détourne des affiches de Mai 685. Ces affiches sorties de l’Atelier populaire de l’École nationale supé-

4. Les illustrations mentionnées dans l'article figurent en annexe.

5. Ces affiches, que nous n'avons pas été autorisé à reproduire ici, sont visibles sur le site pics. agora : [http://pics.agora.eu.org/spip.php?page=recherche\&recherche=Leclerc], consulté le 23 janvier 2012. 
rieure des beaux-arts de Paris (ENSBA) et de l'École nationale supérieure des arts décoratifs (ENSAD) servaient alors de support graphique à la contestation de l'ordre établi (Zaccaria, 2008). Elles prenaient alors position politiquement contre le gouvernement avec des slogans qui sont restés dans les mémoires et dont l'évocation suffit à réactiver les représentations révolutionnaires dans l'imaginaire collectif. Malgré les similitudes entre les affiches de Mai 68 et leur récupération, la présence du logo «E. Leclerc» sert à l'identification des énoncés comme appartenant au genre publicitaire et à orienter le discours vers une finalité commerciale.

Alors que certaines des affiches de Mai 68 évoquent la lutte et le monde ouvrier, chacune des publicités présente deux domaines ${ }^{6}$ qui s'entremêlent : la consommation et la lutte. Ainsi, le domaine de la consommation est figurativisé ${ }^{7}$ par différents éléments iconiques tels que le groupe de consommateurs, le sac de courses, l'alignement des boîtes de conserves pour rappeler les rayonnages des grandes surfaces, le code-barres ou encore le logo E. Leclerc. Sur le plan textuel, «Leclerc», «pouvoir d'achat», «vendre moins cher» et «prix» permettent une autre actualisation du domaine. L'emprunt iconique du poing brandi des affiches, transposé aux publicités, ainsi que la citation modifiée de l'expression de Mai 68, «il est interdit d'interdire», et le slogan «E. Leclerc défend votre pouvoir d'achat», convoquent le domaine de la lutte. La récurrence de ces deux domaines à travers les trois publicités de la campagne amplifie le discours de l'annonceur et fixe son positionnement. La résistance des manifestants est empruntée iconiquement par E. Leclerc dans une perspective discursive consumériste : la transformation des isotopies (Greimas, Courtés, 1993) modifie les valeurs discursives pour que la lutte serve désormais un idéal de consommation.

Dans ce discours publicitaire, l'annonceur s'adresse à l'actant collectif que sont les consommateurs, en employant une structure dialogique repérable par l'utilisation du pronom votre: «Leclerc défend votre pouvoir d'achat», «la hausse des prix oppresse votre pouvoir d'achat». En informant les consommateurs de son opposition envers des mesures politiques et économiques qui iraient à leur encontre, en contestant la hausse des prix, le distributeur montre son engagement à défendre le pouvoir d'achat en les baissant. Or un paradoxe s'installe puisqu'on peut lire sur une publicité qu' «il est interdit d'interdire de vendre moins cher» : cela suppose qu'il serait déjà interdit de vendre moins cher et cela révèle finalement l'interprétation faite par E. Leclerc de la loi Galland.

6. Le domaine est défini selon François Rastier (1987) comme une classe sémantique liée à l'entour socialisé et aux sphères de l'activité humaine.

7. Le discours est figurativisé quand l'objet syntaxique reçoit un investissement sémantique qui permet à l'énonciataire de le reconnaître comme une figure, «sac de courses » signifiant par exemple «consommation» (Greimas, Courtés, 1993, p.147). 
Comment le niveau iconique relaye-t-il le textuel dans la production du sens? Tandis que le slogan «ll est interdit d'interdire » était scandé par les manifestants de 1968 , celui de la publicité se rapporte aux consommateurs de l'énoncé uniquement ${ }^{8}$. E. Leclerc inclut artificiellement les consommateurs dans son discours militant en adoptant la posture d'un porte-parole dont on peut interroger la légitimité, car il semble que le distributeur défende avant tout ses intérêts quand il conteste une décision politique. Il s'agit donc d'un double discours, un discours publicitaire paré d'un discours militant, qui demande à être inséré dans le débat public et qui prétend défendre les consommateurs en faisant passer les profits de l'entreprise après la santé économique des ménages.

Mais à qui ce discours s'adresse-t-il vraiment? Lorsque Michel-Édouard Leclerc commente les décisions gouvernementales sur les questions touchant la grande distribution, il utilise les canaux de l'information (journal télévisé, émission de radio, article de presse ou encore son blog personnel)9. En recourant à la publicité pour aborder ces mêmes questions, il crée une polémique et s'adresse à deux énonciataires opposés discursivement, les consommateurs et les pouvoirs politiques, ces derniers ne manquant d'ailleurs pas de réagirio.

Mettons maintenant la notion de politisation en lien avec le détournement des affiches de Mai 68 dans la campagne publicitaire de E. Leclerc. Le «travail politique » est défini par Paul Bacot comme une "construction sociale d'une représentation du monde fondée sur une problématisation conflictuelle élargie» (Bacot, 1991, p. 86). Pour qu'une réalité soit perçue et donnée à voir comme problématique, cela suppose de «percevoir - et donner à voir - un écart entre ce qui est et ce qui devrait être. C'est ensuite penser que la réduction de cet écart est souhaitable [...] par des actes appropriés [...]. C'est enfin signaler la responsabilité d'une instance [...]» (ibid., p. 87).

Ainsi, dans le discours de E. Leclerc autour du pouvoir d'achat, l'écart se situe entre «la hausse des prix» (ce qui est) et la baisse des prix (ce qui devrait être), écart qui pourrait être réduit si les responsables politiques abrogeaient la loi. Toutes les formes de problématisation ne relèvent pas du «travail politique » mais seulement celles qui élargissent la conflictualité, c'est-à-dire que des controverses jusqu'ici sans relation nécessitent d'être intégrées à une représentation commune. En pointant la responsabilité des pouvoirs publics, l'enseigne instaure ainsi un clivage qui l'oppose à eux.

8. Il s'agit de faire la distinction entre les consommateurs représentés sur le plan iconique par l'annonceur (les consommateurs "énoncifs») et les consommateurs réels à qui s'adresse la publicité (les consommateurs «énonciataires»).

9. [http://www.michel-edouard-leclerc.com]. Consulté le 17 mars 2011 (sauf indication contraire, tous les sites mentionnés dans les notes ont été consultés à cette date).

10. Le 18 février 2005 , le Premier ministre Jean-Pierre Raffarin juge en effet cette campagne «violente et brutale ». Il ajoute que «s'ils [les Centres Leclerc] veulent baisser les prix, ils n'ont qu'à mettre l'argent de la publicité dans la baisse des prix» [http://lci.tf1.fr/economie/2005-02/raffarin-veut-baisser-prix-4877024.html]. 
Alors que le slogan «Il est interdit d'interdire» condensait un projet de société et une représentation politique du monde, celui de E. Leclerc réduit la question du pouvoir d'achat à celle des prix dans une approche exclusivement économique. Par le maquillage de son intérêt commercial en intérêt général, l'enseigne cherche à légitimer son action, notamment quand elle interpelle le pouvoir politique, en produisant un discours qui se voudrait du côté des consommateurs («Leclerc défend votre pouvoir d'achat»).

Le mouvement de Mai 68 renvoyait à une construction sociale où des problèmes de nature différente s'intégraient à une représentation commune, et donc nécessairement conflictuelle. Penser ces problèmes dans un espace délimité (ou dans la Cité, selon les termes de Paul Bacot) grâce à un clivage qui construit des camps opposés (ou partis), constitue un processus de politisation. Or, E. Leclerc dépolitise le discours des événements de Mai 68 dans le sens d'un rétrécissement de la conflictualité (Bacot, 1993), par la transformation et la recontextualisation d'un slogan politique en slogan commercial. Par ailleurs, en détournant les affiches du mouvement de Mai 68 qui décriait la société de consommation (Baudrillard, 1970), l'annonceur ne se contente pas de caricaturer la politique, il récupère son pouvoir mobilisateur en créant ainsi un discours pro-consommation qui s'affranchit de l'idéologie contestataire, lequel s’apparente à un discours publicitaire politisé.

\section{La lutte du pouvoir d'achat continue}

Dans la lignée des emprunts à Mai 68, cette nouvelle campagne sortie pendant l'été 2005 (figures 4 à 7) témoigne des mouvements de la contre-culture de la fin des années 1960, époque durant laquelle une forte contestation sociale se répand à travers de nombreuses manifestations. Les photographies sont des représentations du monde ; elles segmentent ici une réalité des manifestations en construisant narrativement leur déroulement à partir de séquences types : rassemblement, protestation, interpellation. Par ailleurs, elles placent l'énonciataire au cœur de l'action par le truchement d'un cadrage qui focalise les protagonistes que sont les manifestants et les forces de l'ordre. De plus, il apparaît que l'annonceur cherche à diversifier les domaines récupérés en ajoutant symboliquement des pacifistes et des féministes aux travailleurs. Une interprétation de cette hétérogénéité des profils représentés serait de dire que $E$. Leclerc met en place une stratégie de communication recherchant l'identification et l'adhésion d'un maximum de consommateurs.

Alors que la campagne précédente reprenait suffisamment de motifs iconoplastiques pour rappeler les affiches de Mai 68, les photos de cette nouvelle campagne sont des citations qui ne subissent pas de transformations iconiques. Elles sont cependant marquées par une énonciation sous la forme d'incrustations textuelles. Ces incrustations colorées contrastent fortement avec les photos 
en noir et blanc, et opposent discursivement la modernité de E. Leclerc à l'archaïsme des grèves et des luttes passées. Car «Aujourd'hui, la lutte c'est le pouvoir d'achat», et l'émancipation se fait désormais par la consommation et non plus par la contestation sociale et le soulèvement populaire.

Dans la publicité institutionnelle, c'est la mise en discours de la marque qui fait figure de stratégie communicationnelle et, en se détachant du produit, la marque se dématérialise pour devenir concept et rentrer en résonance avec les attentes et les désirs du consommateur. Alors que la «publicité produit» a comme objectif de mettre en valeur les qualités des objets de consommation et de les faire acheter à un public, qui est toujours in fine le consommateur (Hébert, 1993), E. Leclerc ne montre aucun objet; seuls des prix correspondant à des produits génériques sont affichés : shampooing, piles, déodorant, culotte en coton. Dans une campagne publicitaire, le choix de montrer ou de dire répond à un objectif de valorisation de la marque; comment interpréter alors l'association virtuelle des hippies au shampooing, ou encore celle des ouvriers au déodorant? La stigmatisation, encouragée par les clichés et des valeurs dépréciatives, est rehaussée par un discours du paraître qui s'en prend également au mouvement féministe avec la culotte en coton. Les idéologies qui sont à l'origine des mouvements contestataires sont réduites à une marchandise qui s'achète et qui se montre. Ce qui peut se lire avec ironie comme un clin d'œil à l'histoire n'est sans doute qu'une parodie : il sera toujours nécessaire de se procurer des piles pour alimenter le porte-voix, même pour manifester contre la société de consommation; et ces images qui veulent sans doute jouer avec la fibre nostalgique des consommateurs et qui ravivent un esprit de liberté de l'époque, prennent part à l'euphorie partagée.

Mais plus que l'association des produits avec un groupe, c'est l'émergence d'un nouveau sens des photos-publicités qui est à interroger. Ce qui fait le sens publicitaire (des produits, des prix) défait le sens politique : la décontextualisation des photos originales et l'ironie de leur utilisation en contexte publicitaire pourvanter des gammes de produits génériques à bas prix agissent comme une dépolitisation de la représentation du mouvement historique, dans le sens où un processus d'éloignement des valeurs, par rapport à l'univers perçu comme spécifiquement politique, est engagé (Arnaud, Guionnet, 2005). Dans un éternel recyclage des pratiques sociales, la publicité absorbe la critique qui lui est faite à travers la critique de la société de consommation, pour se revaloriser dans un métadiscours.

\section{Intermarché et l'intérêt général}

L'aspect du politique amenant des questions sur le bien commun et l'intérêt général, l'analyse d'une autre publicité (Intermarché en 2008) permet également de réfléchir aux frontières du discours publicitaire. En récupérant chez 
son concurrent historique ${ }^{11}$ la thématique de la lutte de prix et de Mai 68 , Intermarché utilise les mêmes artifices pour retourner le discours-source contre E. Leclerc. La publicité ${ }^{12}$ sortie à l'occasion du très médiatisé $40^{\mathrm{e}}$ anniversaire de Mai 68 emprunte les codes iconiques déjà utilisés par E. Leclerc : sur un fond gris se détache une étiquette sur laquelle est apposé un prix de vente, et dont le trou béant en son milieu indique qu'un pavé, à terre, l'a transpercée; la publicité s'interprète comme l'illustration figurative de l'expression «casser les prix»13.

Les guillemets qui encadrent la phrase «Nous, on n'a pas arrêté la lutte» sont le marqueur de l'énonciation énoncée ${ }^{14}$ par Intermarché. Alors que le slogan «on n'a pas arrêté la lutte » permettrait à Intermarché de clamer aux consommateurs son refus de la vie chère, l'antéposition du pronom «nous » marque une distinction de l'Autre, notamment E. Leclerc; ce "nous» mis en exergue par la couleur rouge, reprise chromatique du logo, s'oppose à un «ils» ou un «vous» et crée un rapport dialogique entre Intermarché et ses consommateurs, d'une part, et entre Intermarché et E. Leclerc, d'autre part. Par un jeu métadiscursif, le distributeur prend acte de la campagne de son concurrent. Double discours, donc, qui pourrait signifier que la génération de 1968 n'aurait pas continué à lutter pour ses idées, mais surtout que E. Leclerc n'aurait pas non plus continué la lutte des prix. En même temps qu'Intermarché présente son engagement auprès de ses clients « contre la vie chère », le distributeur signale son objectif de poursuivre une lutte concurrentielle.

Cependant, le slogan historique « tous unis contre la vie chère » expose l'annonceur à contribuer à l'intérêt général en contrant une augmentation des prix, et permettre à tous une consommation à faible coût. La posture d'Intermarché consiste à faire passer l'intérêt général avant le sien, ce qui esquisse, d'après Dominique Quessada (1999), l'évolution et le débordement des discours d'entreprises privées sur le champ politique dans les sociétés néolibérales. Même si, comme le dit l'expression, l'union fait la force, on se demande comment les consommateurs ont de leur côté un quelconque pouvoir pour faire baisser les prix et comment imaginer cette union avec l'entreprise. Cette union apparente poserait le consommateur en militant et légitimerait sa «consommation engagée » (Granier, 2008) comme un acte de rébellion. Rien n'est moins sûr si l'on suit le propos de Paul Ariès (2003) cité par le Groupe Marcuse (2010) : «La publicité a tué l'esprit de révolte après avoir asséché l'imaginaire.»

11. Le groupe Intermarché est né d'une scission d'avec Leclerc en 1969. L'enseigne EX voit le jour, avant d'être abandonnée au profit d'Intermarché en 1973.

12. Conçue par l'agence Publicis Conseil, l'annonce paraît le dimanche 4 mai 2008 dans le quotidien Le Parisien.

13. Cette affiche est visible sur le site de Stratégies: [http://www.strategies.fr/actualites/marques/ r48467W/mai-68-c-est-folklo.html].

14. L'énonciation énoncée est un simulacre énonciatif, une représentation discursive de l’instance d'énonciation (Greimas, Courtés, 1993, p. 125). 


\section{Du discours publicitaire au discours politique : disparition des frontières}

Les discours publicitaires précédemment analysés montrent la construction d'une «conflictualité élargie non politicienne » (Bacot, 1993, p. 119) autour de la question du pouvoir d'achat, et font coexister différents acteurs qu'un clivage matriciel répartit en camps opposés : d'un côté les enseignes, de l'autre les pouvoirs publics à qui on demande d'intervenir pour permettre une baisse des prix. En prétendant défendre le pouvoir d'achat, les annonceurs s'assignent une mission «politique » et remettent en question la capacité des «politiciens » professionnels à satisfaire l'intérêt général, ce qui contribue à créer une opposition plus générale entre la société (enseignes alliées aux consommateurs, prétendus avoir les mêmes intérêts) et les acteurs de la scène politique.

La thèse de Dominique Quessada apporte un complément pour analyser les ambitions totalisantes du discours publicitaire même s’il n'emploie pas la même terminologie et ne distingue pas le politique (au sens noble de la gestion des affaires de la Cité, policy chez les Anglo-Saxons) de la politique (souvent entendue au sens péjoratif de la «lutte politicienne», politics). En effet, selon lui, «la publicité constitue le discours qui dissout toutes les frontières» (1999, p. 85) et en cela, il produit de l'incertitude « entre un discours politique (énoncé dans le cadre politique traditionnel) et un autre (énoncé désormais par la publicité)» (ibid.). Les messages publicitaires des enseignes déplacent ainsi les frontières du clivage, et en visant le bien commun, elles défendent une conception «noble » de la politique à l'opposé d'une version politicienne, censée tromper la société et entraver la liberté. La publicité serait donc «un discours politique ne relevant pas de l'ordre du politique» (ibid.), et cela est notamment rendu possible par le flou qu'elle entretient entre les notions de public et de privé, et d'information et de communication.

Les publicités institutionnelles comme celles de E. Leclerc ou d'Intermarché sont très illustratives à ce sujet : il s'agit d'informer-communiquer sur l'apport de l'entreprise à la société et donc de son souci pour l'intérêt général qui, dans le discours de E. Leclerc, se décline en pouvoir d'achat:

La question du pouvoir d'achat me passionne. Je n'arrive pas à imaginer qu'on puisse parler de bien-être, sans un minimum d'aisance matérielle. C'est une question politique : le niveau de pouvoir d'achat détermine pour les citoyens un état de liberté d'épargner, d'acheter, d'arbitrer dans le choix des produits, y compris pour les plus démunis. (Leclerc, 2004b; nous soulignons)

L'interprétation du mot liberté est ici assez réductrice : elle devient celle d'acheter, celle de choisir... parmi tous les produits présentés par la marque; «la liberté devient le maître mot par lequel le citoyen est transformé idéologique- 
ment en consommateur» (Quessada, 1999, p. 27). Ainsi s'opère «le déplacement généralisé du centre de gravité du politique vers l'économique dans les sociétés contemporaines » (ibid.). Le distributeur tient donc à faire reconnaître son action pour le bien général et réclame le droit de faire entendre sa voix; il peut, à travers la publicité, interpeller le président qui n'a pas tenu ses promesses ou manifester son désaccord vis-à-vis des lois réglementant le marché («il est interdit d'interdire de vendre moins cher»).

Selon Michel-Édouard Leclerc, la politique est un terrain à investir en tant que citoyen; participer à la vie politique est un devoir et bien qu'il reconnaisse ne pas avoir la légitimité du scrutin, il revendique son « expertise de chef d'entreprise» fondée sur la base des réussites commerciales et regrette qu'elle ne soit pas reconnue dans le champ politique. La publicité serait un moyen parmi d'autres possibles pour exposer les idées et «forcer l'écoute [des] élus» (Leclerc, 2004a). Les propos tenus sont partagés, selon lui, par les citoyens qui leur donnent de ce fait «une force supplémentaire» (ibid.). Il invite par ailleurs les chefs d'entreprise à le suivre parce que finalement, c'est une recette gagnante. En effet, en 2005, la loi Galland connaît un début de réforme et autorise la baisse des prix des grandes marques. Elle « doit contribuer à dynamiser la concurrence pour faire baisser les prix dans les grandes surfaces» (Portail du gouvernement, 2007). L'entreprise dite citoyenne prétend fabriquer de l'intérêt général, elle « instrumentalise la citoyenneté au service de l'amélioration de l'image des entreprises et de leur capacité d'intervention dans la sphère politique »15 (Quessada, 1999, p. 90). Si l'acteur politique a montré ses limites pour défendre le pouvoir d'achat, les distributeurs, qui incarnent la puissance d'agir, montrent qu'ils peuvent le faire, une fois affranchis des entraves d'ordre politique telle la loi Galland.

Le trouble entre le politique-politique et le politique-publicitaire, selon les termes de Dominique Quessada, est entretenu également par l'emprunt que fait le discours publicitaire à la rhétorique politique : «La publicité n'efface pas la politique, au contraire, elle l'affiche. $»^{16}$ Nous avons vu de quelle manière E. Leclerc et Intermarché dépolitisent les images contestataires des mouvements sociaux en les détournant et, à travers ces détournements, comment ces entreprises exposent leur engagement à défendre le pouvoir d'achat en adoptant une position militante. Leur discours publicitaire reprend du discours politique son aspect fondamental, c'est-à-dire l'action sur la société, pour la remettre au service de ses propres intérêts, leur but étant d'attaquer le monopole de la politique «comme seul mode d'organisation et de régulation de la société» (ibid., p. 99). Les luttes sociales du passé convoquées par le discours

15. R. Lesgards, «L'entreprise en mal de citoyenneté », Le Monde diplomatique, juin 1996, cité par Quessada, 1999, p. 90.

16. Y. Jeanneret, E. Souchier, «Publicité et politique », Le Monde diplomatique, décembre 1994, cité par Quessada, 1999, p. 93. 
de E. Leclerc sont présentées comme archaïques, démodées, car «aujourd’hui, la lutte est pour le pouvoir d'achat ». L’idée étant que la politique, représentée par le mouvement de Mai 68 ou d'autres mouvements sociaux de l'époque, n'a pas su apporter le bonheur aux citoyens, contrairement à ces entreprises qui parviendraient, par le biais de la consommation, à apporter un nouveau bonheur pour tous, "y compris les plus démunis» (Leclerc, 2004b). Ainsi l'affiche détournée de Mai 68, dans laquelle le poing est brandi comme symbole révolutionnaire et porté par les boîtes de conserves, devient un appel à la consommation. Le discours de $\mathrm{E}$. Leclerc caricature donc la politique et récupère sa force mobilisatrice.

La publicité utilise le langage et les mots de la politique, elle détourne le politique, et finit par le remplacer. Elle exploite un désir de changement et des convictions qu'elle sait mettre au service de ses intérêts, en se présentant comme le changement lui-même. (Quessada, 1999, p. 101)

Ainsi, des adjonctions produisent des déplacements de sens : «tous unis contre la vie chère", "promettre la hausse du pouvoir d'achat c'est permettre la baisse des prix», "la croissance oui sauf celle des prix» ou encore "il est interdit d'interdire de vendre moins cher». Ces énoncés mettent en question la loi Galland, et à travers elle le politique lui-même pour mieux se substituer à lui. Le discours publicitaire des enseignes, en prenant en charge le discours collectif, modifie l'espace dans lequel ce dernier s'inscrit. Il ne s'agit plus d'espace public qui renvoie au politique, mais d' «espace commun», comme le propose Quessada. La publicité constitue finalement un détournement, voire une parodie du discours politique, mais elle devient surtout « un discours politique [...] qui se présente comme une alternative au discours politique traditionnel» (ibid., p.101).

Si la division en camps ou partis est au cœur du politique, ces publicités renvoient à l'unification, à l'idée qu'on peut harmoniser la société jusqu'à expulser tous vestiges de division. À travers des slogans comme «tous unis contre la vie chère » ou "Leclerc défend votre pouvoir d'achat», le discours publicitaire réunit l'entreprise, devenue citoyenne, aux consommateurs-citoyens par l'équivalence faite entre pouvoir d'achat et intérêt général. L'entreprise est ainsi capable d'apporter le bonheur et la liberté à travers la consommation, tout ce qui peut entraver cette alliance heureuse étant imposé par des lois régulatrices de l'économie, donc des politiques. Ainsi, la publicité construit ici un discours politique qui se veut comme un recours contre le discours politique traditionnel, qu'elle a contribué à vider de son sens :

Elle génère ainsi la croyance qu'elle est une force organisationnelle supérieure à la politique, parce qu'elle contiendrait un projet global plus souple, plus homogène et plus collectif. Elle réalise alors le projet du discours politique mieux que le discours politique lui-même. (Ibid., p. 102). 
Lorsque ce type de discours publicitaire avance sur de nouveaux terrains et en particulier le terrain politique, il ne transgresse pas de frontières - car cellesci continueraient à exister même si elles étaient franchies - mais il les fait disparaître, d'où le trouble, l'incertitude, la difficulté à identifier des espaces différenciés comme ceux du privé et du public ou encore ceux de l'information et de la communication. L'évolution de ce discours publicitaire, qui tend à devenir un discours sans frontières, invite donc à croiser les regards, à convoquer la pluridisciplinarité pour mieux saisir les enjeux actuels. Ce n'est pas seulement une affaire des sciences de l'information et de la communication mais aussi, peut-être, une question à aborder dans le champ de la science politique.

\section{Références}

ACHACHE Gilles, 1990, "La communication, déclin ou extension de la politique?", Esprit, n¹64, septembre, p.145-151.

ARIÈs Paul, 2003, Putain de ta marque! La pub contre l'esprit de révolte, Villeurbanne, Golias.

ARnAud Lionel, Guionnet Christine éd., 2005, «Introduction», Les frontières du politique. Enquêtes sur les processus de politisation et de dépolitisation, Rennes, Presses universitaires de Rennes, p. 11-25.

BAcot Paul, 1991, «Une représentation politique du sida. Quelques modes discursifs au PCF», Mots. Les langages du politique, n²6, p. 85-103.

- 1993, "Conflictualité sociale et geste électoral. Les formes de politisation dans les lieux de vote ", Revue française de science politique, vol.XLIII, nº 1, p.107-135.

BAUDRILLARD Jean, 1970, La société de consommation : ses mythes, ses structures, Paris, Denoël.

E. LeCLerC (Enseigne), 2008, «E. Leclerc et la défense du pouvoir d'achat», Mon pouvoir d'achat, en ligne [http://www.mon-pouvoir-dachat.com/defense-pouvoirachat.html], consulté le 17 mars 2011.

EliASO PH Nina, 1998, Avoiding Politics. How American Produce Apathy in Everyday Life, Cambridge, Cambridge University Press.

FCA (Fédération des enseignes du commerce associé), 2011, «E. Leclerc creuse l'écart en 2010 », FCA, en ligne [http://www.commerce-associe.fr/fevrier-2011-c-3034. php\#leclerc], consulté le 17 mars 2011.

GRANIER Corinne, 2008, «La publicité politisée. Éloge de la transparence, ellipse de la responsabilité », Les enjeux de l'information et de la communication, année 2008 , p. 10-19.

GreImAS Algirdas Julien, Courtés Joseph, 1993, Sémiotique. Dictionnaire raisonné de la théorie du langage, Paris, Hachette.

Groupe Marcuse, 2010, De la misère humaine en milieu publicitaire. Comment le monde se meurt de notre mode de vie, Paris, La Découverte.

HAMIDI Camille, 2006, "Éléments pour une approche interactionniste de la politisation », Revue française de science politique, vol. LVI, nº 1, p. 5-25. 
HÉBERT Nicole, 1993, L'entreprise et son image. La publicité institutionnelle, pourquoi, comment?, Paris, Dunod.

JEUdY Bruno, 2008, «En baisse dans les sondages, Sarkozy repart sur le terrain», Le Figaro.fr, en ligne [http://www.lefigaro.fr/politique/2008/01/20/0100220080120ARTFIG00052-la-popularite-de-sarkozy-en-baisse.php], consulté le 17 mars 2011.

LeCLeRC Michel-Édouard, 2004a, "Convictions. P comme... Politique», De quoi je me M.E.L, la tribune de Michel-Édouard Leclerc, en ligne [http://www.michel-edouardleclerc.com/blog/m.e.l/convictions/p/index.php], consulté le 17 mars 2011.

- 2004b, "Défendre le pouvoir d'achat», De quoi je me M.E.L, la tribune de MichelÉdouard Leclerc, en ligne [http://www.michel-edouard-leclerc.com/blog/m.e.l/ engagements/defendre-le-pouvoir-dachat/defendre-le-pouvoir-dachat.php], consulté le 17 mars 2011.

MAURIAC Laurent, NATHAN Hervé, 2004, «Pouvoir d'achat : la polémique en hausse», Libération.fr, en ligne [http://www.liberation.fr/evenement/0101478674-pouvoird-achat-la-polemique-en-hausse], consulté le 17 mars 2011.

Perona Mathieu, 2004, "But et enjeux de la loi Galland », Les Dossiers du Net, en ligne [http://www.dossiersdunet.com/spip.php?article245], consulté le 17 mars 2011.

PORTAIL DU GOUVERNEMENT, 2007, «Réforme de la loi Galland», en ligne [http://www. gouvernement.fr/gouvernement/reforme-de-la-loi-galland], consulté le 17 mars 2011.

QueSSADA Dominique, 1999, La société de consommation de soi, Paris, Verticales.

RASTIER François, 1987, Sémantique interprétative, Paris, PUF.

ZACCARIA Diego, 2008, L'affiche, paroles publiques, Paris, Textuel. 




Figure 1. Campagne E. Leclerc @ 2004

Monsieur le Président, promettre la hausse du pouvoir d'achat, c'est permettre la baisse des prix. Visiblement, c'est mal parti.

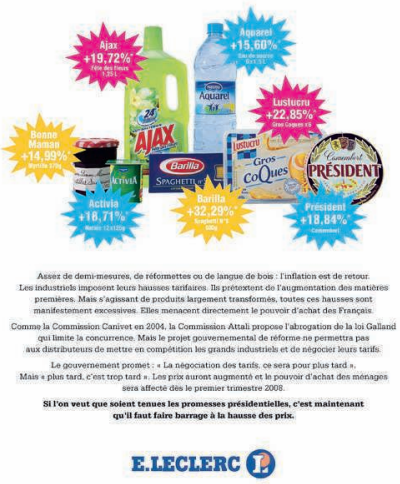

Figure 2. Campagne E. Leclerc @ 2007



Figure 3. Campagne E. Leclerc @ 2007 


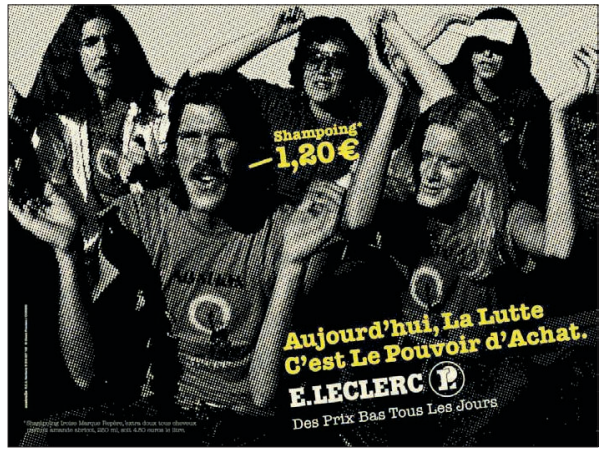

Figure 4. Campagne E. Leclerc () 2005

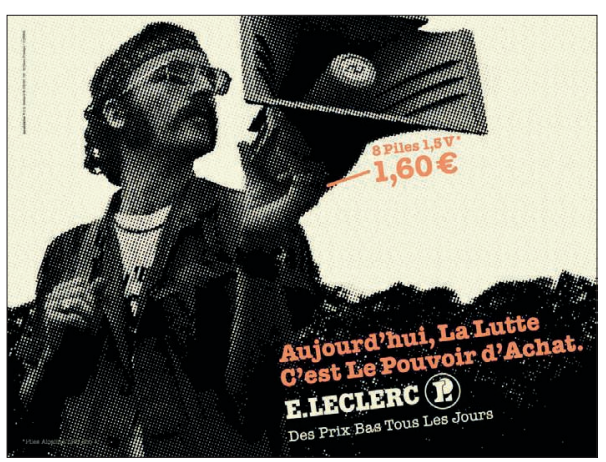

Figure 5. Campagne E. Leclerc (C) 2005

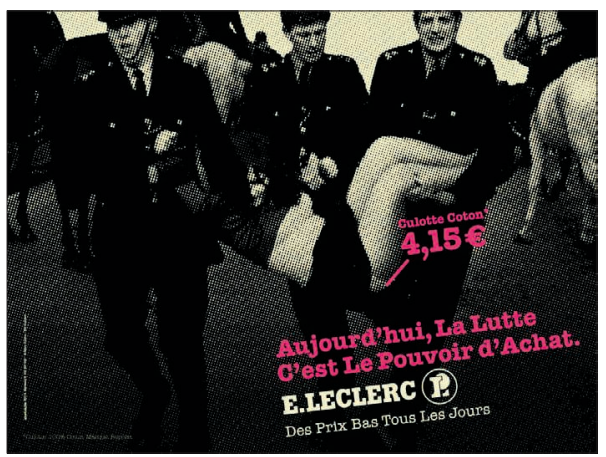

Figure 7. Campagne E. Leclerc $@ 2005$

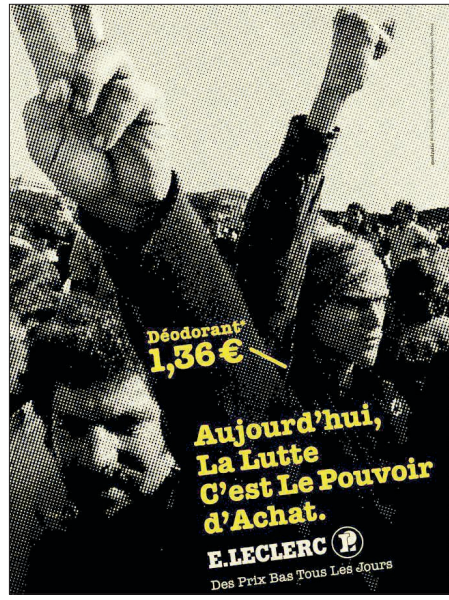

Figure 6. Campagne E. Leclerc (C) 2005 